\title{
Covering problems and Influence maximization
}

\author{
Gyöngyvér Vass \\ Institute of Informatics \\ University of Szeged \\ Szeged, Hungary \\ Vass.Gyongyver@stud.u-szeged.hu
}

\author{
Boglárka G.-Tóth \\ Institute of Informatics \\ University of Szeged \\ Szeged, Hungary \\ boglarka@inf.szte.hu
}

\begin{abstract}
Influence maximization is a very popular problem in the social sciences. It seeks a given number of seed points (vertices) in a network to maximize the number of influenced vertices starting from the seeds. Influencing may occur trough the edges, which indicate the connection between people (vertices). There are different ways to define influence, but up to our knowledge, finding the seeds from which maximal influence can be reached is a difficult task in general.

Coverage models belong to facility location problems, where centers are to be located such that the covered demand points (vertices in a graph within a given distance) are maximized. These models are motivated by problems where some services are only available within a fixed radius, like ambulances or fast food delivery. These problems are solvable for large graphs, and our long term aim is to use the most appropriate and/or adjusted covering models for solving influence maximization problems. As a first step, in this paper, we compare influence maximization and coverage models and analyze their differences.
\end{abstract}

As we will show, there are many similarities between the models, however, the main difference is that covering is a static action while influencing is dynamic. We show when this difference can be resolved, and how different are the results when not.

\section{Categories and Subject Descriptors}

G.2.2 [Mathematics of Computing]: Graph TheoryNetwork problems; G.1.6 [Mathematics of Computing]: Optimization

\section{General Terms}

Theory, application

\section{Keywords}

Covering models, Influence maximization, Mixed Integer Programming

\section{INTRODUCTION}

The influence maximization problem explores that from a given seed point how many other individuals can be reached by the information in a social network. The transmitting of the information and the terms of the transmitting can be different, but usually, the capability of the influencing is impressed by the weight or strength of the edge between two points. Influence maximization is also known as information diffusion. For a survey on the subject see $[2,3]$.

In facility location, the covering problem explores the point or points that can cover other points in a demand network within a given covering distance. For a good introduction to facility location problems, see [1].

Up to the knowledge of the authors, these models have not yet been compared in the literature, thus results in this paper are completely novel. As covering models are well studied and efficient algorithms exist to solve large scale problems, it is interesting to investigate if the influence maximization problem (or its slight modification) can be solved by any approach made for covering models. Thus, we aim to compare influence maximization and covering models as a first step.

\section{INFLUENCE MAXIMIZATION}

Formally, the influence maximization problem can be defined as follows. There is a simple graph $G=(V, E)$, where the vertices of the graph $v \in V$ represent the individuals and the edges represent the connections between them. Hereafter we can regard as $|V|=n$ and $|E|=m$, thus, in the social network there are $n$ people, and $m$ connections between them. We assign a weight to each edge $e \in E$, which will give us a probability: $f(e): E \rightarrow[0,1]$ the probability that information can spread trough a given edge $e$, also it can be seen as the strength of the relationship.

For the influence maximization model, there is a set $S \subset V$, from which the information will start, called seed set. The cardinality of the set $S$ is fixed, we will denote it with $s$ $(s<n)$. This gives us a general diffusion model, where the diffusion function is: $\sigma(S): V \rightarrow[s, n]$, that is, the number of vertices influenced by the seed set $S$. We seek for the seed set maximizing the influence, i.e. $\max _{S \subset V} \sigma(S)$. 
In the general model described above the processing of the influencing can be differently modeled. The most known models are the linear threshold model and the independent cascade model, see [3], however for our purposes the so-called triggering model is the most appropriate.

\subsection{Triggering Model}

In the triggering model, for each $v \in V$ we independently choose a random $T_{v}$ triggering set according to some distribution over subsets of its incoming neighbours. The $T_{v}$ set is a subset of the neighbours of vertex $v$. At the beginning of the process, the seed set $S$ will be active. An inactive point becomes active at time instant $t$, if any element of the selected triggering set $T_{v}$ becomes active at time instance $t-1$. Formally, for $v \in V \backslash S$ if exists $v^{\prime} \in T_{v}$ such that $v^{\prime} \in A_{t-1}$, then $v \in A_{t}$.

It can be seen that in this model, probability and threshold, used in the threshold model and the independent cascade model [3], are replaced by an influencer set that represents the way information is spread. This also means that this model is deterministic from the point where the triggering sets are chosen.

We have designed a mathematical model for this problem so that it can be solved with a Mixed Integer Programming (MIP) solver. As a parameter, we need the maximum number of steps of the influencing process, $t_{\max }$. It is not known beforehand but can be taken as the diameter of the graph, which is a good upper bound for the run time. The rest of the data is the graph itself and the triggering set for each vertex $T_{j}$, which includes a subset of the neighbours of $j$ and also $j$.

Decision variables:

$Z_{j t}= \begin{cases}1, & \text { if point } j \text { is active at step } t \\ 0, & \text { otherwise }\end{cases}$

$$
\begin{array}{ll}
\max & \sum_{j} Z_{j t_{\max }} \\
\text { s.t. } & \sum_{j} Z_{j 0}=s \\
& \sum_{i \in T_{j}} Z_{i t} \geq Z_{j, t+1} \quad \forall j \in V, 0 \leq t<t_{\max }
\end{array}
$$

Variables $Z_{j t_{\max }}$ gives us the resulting influenced nodes after $t_{\max }$ steps, thus the objective function is to maximize their sum. Condition (2) sets the number of initial seeds to $s$, while in (3) the influencing is defined. Namely, a vertex $j$ is influenced if its neighbours in $T_{j}$ are influenced. By maximizing the influence, the objective guarantees that $Z_{j, t+1}$ will always take value 1 if the left-hand side of (3) allows it, so no lower bounding condition on $Z_{j, t+1}$ is necessary.

\section{COVERING PROBLEMS}

Covering problems belong to the field of facility location. We interpret it on a network and consider vertices as demand points. Our goal is to place facilities or service units at some vertices of the network that can cover as many demand points as possible. Distance between two vertices is defined by the length of the shortest path between the two points, where edge lengths may be given or considered unit length. The covering distance or covering radius is also given to the problem, which tells us in what distance the new facility can cover the demand points. In real life, it may depend on the "size" of the facility, for example, in hospitals, its floor area affects how many beds can be laid out and thus how many patients can be accommodated, or on the maximal distance a service can be realized (ambulance, fast food delivery for example). Formally, we consider a simple graph $G=(V, E)$ with the usual notation. The concept of coverage can be defined as follows. Our goal is either to maximize the covered demand by a fixed number of companies or to cover the entire network minimizing the number of centers, or building costs. We will only discuss the first model, called maximal covering.

\subsection{Maximal Covering}

In this model, we aim to maximize the number of covered demand points locating a fixed number of facilities at the vertices of the network. The number of facilities to be located is $s$, and the covering radius $R$ should also be known for the problem. Formally, we can write the model as follows.

Parameters:

$a_{i j}= \begin{cases}1, & \text { if point } i \text { can cover point } j, \text { i.e. } d(i, j) \leq R \\ 0, & \text { otherwise }\end{cases}$

Decision variables:

$X_{j}= \begin{cases}1, & \text { if a company at point } j \text { is located } \\ 0, & \text { otherwise }\end{cases}$

$Y_{j}= \begin{cases}1, & \text { if point } j \text { is covered } \\ 0, & \text { otherwise }\end{cases}$

Having these parameters and decision variables, the objective function and constraints can be written in the following way.

$$
\begin{array}{ll}
\max & \sum_{j} Y_{j} \\
\text { s.t. } & \sum_{j} X_{j}=s \\
& \sum_{i} a_{i j} X_{i} \geq Y_{j} \quad \forall j
\end{array}
$$

The objective of the model described in (4) is to maximize coverage, which is the number of covered demand points. Constraint (5) ensures that exactly $s$ facilities are located. Each demand point $j$ has a constraint that ensures that demand point $j$ is covered only if there is a facility located within the given distance, see (6). Knowing the coverage distance, we can determine the locations which could cover the demand point $j$. 
Table 1: Comparison of the models

\begin{tabular}{|l|c|l|}
\hline Covering problem & & Influence maximization \\
\hline graph of demand points and roads & $\boldsymbol{\checkmark}$ & social network (graph) \\
\hline we want to locate facilities at demand points (vertices) & $\boldsymbol{\checkmark}$ & we want to find the seed points (vertices) \\
\hline cover other demand points & $\boldsymbol{V}$ & influence other points \\
\hline $\begin{array}{l}\text { Covering: a demand point is covered if there is a company } \\
\text { for which their distance is less than the coverage distance }\end{array}$ & $\boldsymbol{X}$ & $\begin{array}{l}\text { Influencing: a point is influenced, if any neighbour could } \\
\text { influence it with the probability of the edge }\end{array}$ \\
\hline static, only facilities can cover & $\boldsymbol{X}$ & dynamic, anyone can influence \\
\hline edge weights are distances & $\boldsymbol{X}$ & edge weights are probabilities \\
\hline deterministic & $\boldsymbol{X}$ & stochastic \\
\hline
\end{tabular}

\section{COMPARISON OF THE MODELS}

Let's first look at the similarities and differences between the two models in general, summarized in Table 1.

Both models work with simple graphs and choose vertices based on the weights of the edges. None of the models need to add any new point, just choose one or more from the existing vertices (these are going to be the seeds or centers), and either spread the information to the neighbours or be the location for the new facilities. The aim of both models is to reach as many points as possible. However, the definition and the way the seeds reach the other vertices are different. We count a point as covered if there is at least one facility where their distance, that is the total weight of the shortest path between the demand point and the new company, is less than the given covering distance. We count a point as influenced if at least one of its neighbours influence it with the edge probability between them. Thus, one of the largest difference is that the maximal covering problem is static, while the information diffusion is dynamic.You: Besides these, another difference is, that in the covering model only the new facility or facilities can cover the demand points, while in the influence maximization problem every influenced point can further influence its neighbours. In the first case, there is a concrete distance, whilst in the second case, there are only probabilities for the spreading, where the spreading can take any number of steps. It also means that covering is deterministic, as the coverage is always the same, while information diffusion is stochastic, as every time we generate random values to simulate the spreading.

\subsection{Comparison of a modified triggering model and the maximal covering model}

A modified triggering model may provide a solution to overcome these differences. To repeat, in the triggering model, each point independently selects a random set (triggering set $T_{j}$ ) based on the distribution of subsets of the neighbours. If one point in $T_{j}$ becomes active, so does the point $j$. This will result in two types of edges, "live" and "blocked" as they belong to the triggering set or not. Now, considering the sub-graph with only the "live" edges, and restricting the run time of the triggering model to $R$ number of steps, the problem equivalent to the maximal covering problem on the sub-graph with covering radius $R$.

Therefore, we propose to modify the triggering model by setting a maximum time for the spread of information, that is the number of steps.
The modified triggering model can be solved by the optimization problem in (1-3) where the maximum number of steps is set to $R$, and $T_{j}$ is the triggering set for each vertex, being the set of all the neighbours of $j$.

Let us show that the model (1-3) is equivalent to the maximal covering model (4-6). As a first step, let us rewrite the condition (6) without the parameter $a_{i j}$, only relying on $d(i, j)$, the distance of vertices $i$ and $j$.

$$
\sum_{i \in V: d(i, j) \leq R} X_{i} \geq Y_{j} \quad \forall j
$$

Now, in order to show the equivalence, we write the models side by side, where in each line the corresponding parts are given.

$$
\begin{aligned}
& \max \sum_{j} Y_{j} \quad \max \sum_{j} Z_{j R} \\
& \text { s.t. } \sum_{j} X_{j}=s \quad \text { s.t. } \quad \sum_{j} Z_{j 0}=s \\
& \sum_{j \in V: d(i, j) \leq R} X_{j} \geq Y_{i} \quad \sum_{i \in T_{j}} Z_{i t} \geq Z_{j, t+1} \\
& \forall i \quad \forall j, 0 \leq t<R
\end{aligned}
$$

For the variables, $Y_{j} \equiv Z_{j R}$, and $X_{j} \equiv Z_{j 0}$, which makes the equivalence in lines (8-9) obvious. In order to see that the conditions in (10) are defining the same constraints, let is rewrite the influence condition as follows.

Aggregating $\sum_{k \in T_{i}} Z_{k, t-1} \geq Z_{i t}$ and $\sum_{i \in T_{j}} Z_{i t} \geq Z_{j, t+1}$ we obtain

$$
\sum_{k \in T_{i}} \sum_{i \in T_{j}} Z_{k, t-1} \geq Z_{j, t+1}
$$

and following this for all the $R$ steps, the condition becomes

$$
\sum_{i_{0} \in T_{i_{1}}} \sum_{i_{1} \in T_{i_{2}}} \cdots \sum_{i_{R-1} \in T_{j}} Z_{i_{0}, 0} \geq Z_{j, R}
$$

Now, if we take into account, that the above sums are only adding those $Z_{i_{0}, 0}$, where $i_{0} \in T_{i_{1}}, i_{1} \in T_{i_{2}}, \ldots, i_{R-1} \in T_{j}$, the requirement is actually the same as $d\left(i_{0}, j\right) \leq R$. There- 
fore the condition can be written as

$$
\sum_{j \in V: d(i, j) \leq R} Z_{i, 0} \geq Z_{j, R} \quad \forall j
$$

which is equivalent to (7) changing the names of the variables.

\subsection{Computational comparison of the models}

To compare the models we implemented them in AMPL, a mathematical programming language. We use (1-3) to define the triggering model and (4-6) for the maximum covering model. The problems were solved by the commercial optimization solver CPLEX.

Let us show the results on an example graph drawn in Figure 1. We run the maximum covering problem on this graph and empirically showed that the same result is obtained when we include all neighbours in the triggering sets.

In order to generate the triggering sets for the general model, we use a selection rate $r$, which gives the proportion of the neighbours to be selected to the triggering sets. If the selection rate $r=1$, we take every neighbours of each vertex to its triggering set. If $r<1$, we select randomly from the neighbouring edges until we have the required number of edges in the triggering sets.

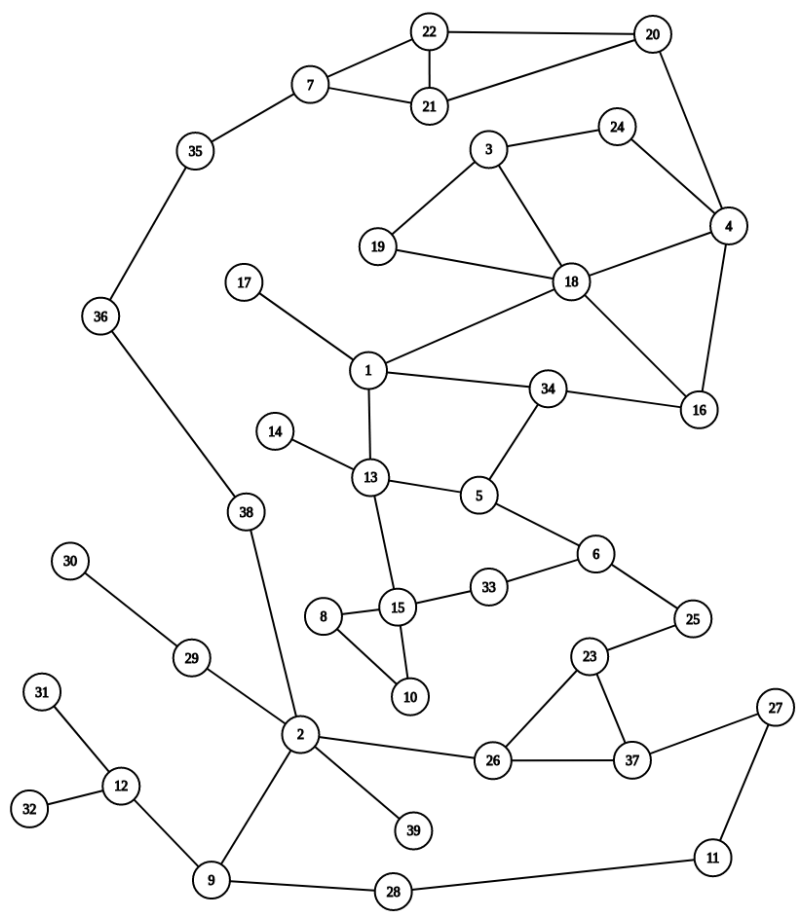

Figure 1: Example graph with 39 nodes.

We summarized the results in Table 2. We set the selection rate from 0.3 to 1 per 0.1 . First, we set the cardinality of the seed set $|S|$ to 1 and the run time $R$ to 4 , and reported the average of the objective value after 50 runs together with the standard deviation for each selection rate. Not surprisingly, decreasing the rate, the average number of influenced points is also decreasing. The standard deviation is rather high, which can be explained by the structure of the graph. There
Table 2: Computational result for the modified triggering model with different number of seeds $|S|$ and run time $R$.

\begin{tabular}{c|cc|cc|cc} 
& \multicolumn{2}{|c|}{$|S|=1, R=4$} & \multicolumn{2}{|c}{$|S|=2, R=3$} & \multicolumn{2}{|c}{$|S|=3, R=2$} \\
Rate & Avg & Dev & Avg & Dev & Avg & Dev \\
\hline 0.3 & 9.1 & 2.0 & 14.3 & 2.0 & 15.8 & 1.9 \\
0.4 & 11.1 & 2.4 & 15.7 & 1.8 & 17.6 & 1.6 \\
0.5 & 14.8 & 2.0 & 20.6 & 2.2 & 20.7 & 1.7 \\
0.6 & 16.6 & 2.2 & 24.4 & 2.5 & 24.8 & 2.0 \\
0.7 & 17.9 & 1.7 & 26.7 & 2.2 & 26.1 & 1.6 \\
0.8 & 20.8 & 0.8 & 32.9 & 2.8 & 29.8 & 1.4 \\
0.9 & 22.0 & 0.0 & 35.9 & 0.5 & 31.9 & 0.3 \\
1.0 & 22.0 & 0.0 & 36.0 & 0.0 & 32.0 & 0.0
\end{tabular}

are long chains in the graph, like the paths 2-7 or 9-37, from which any edges become blocked, the influenced set can change easily. This also means that the seed is very unstable between the different runs, even for high selection rates.

In the next columns we report the results for $|S|=2$ and $R=3$, and also for $|S|=3$ and $R=2$. The obtained results are quite similar as before, although we can see that the deviation of the results is smaller for the last case $(|S|=3$, $R=2$ ). From these results, we can see that for high selection rates the models give quite similar results in terms of the objective value, but we have seen the seed sets are quite different except for rate 1 .

\section{CONCLUSIONS}

It has been intuitively shown that maximal covering and influence maximization deal with a similar problem. The research revealed to us what similarities and differences are. In both cases, we start from a very similar problem and have a similar goal. However, we also show that one of the largest differences is the static nature of facility location and the dynamic nature of information diffusion. We have seen a solution to this by using the modified triggering model. It is planned to compare the results of the models for a large set of networks and to analyze more information diffusion models in the near future.

\section{ACKNOWLEDGMENTS}

The project was supported by the European Union, co-financed by the European Social Fund (EFOP-3.6.3VEKOP-16-2017-00002).

\section{REFERENCES}

[1] M. S. Daskin. Network and Discrete Location: Models, Algorithms and Applications. John Wiley and Sons, New York, 1995.

[2] V. Homolya. Analysis of information diffusion in networks (in hungarian), 2017. Hungarian Conference for Students (OTDK).

[3] G. D. Nittis and N. Gatti. How to maximize the spread of social influence: A survey. CoRR, abs/1806.07757, 2018. 